Document downloaded from:

http://hdl.handle.net/10251/60812

This paper must be cited as:

Prats-Montalbán, JM.; Sanz Requena, R.; Marti Bonmati, L.; Ferrer, A. (2014). Prostate functional magnetic resonance image analysis using multivariate curve resolution methods. Journal of Chemometrics. 28(8):672-680. doi:10.1002/cem.2585.

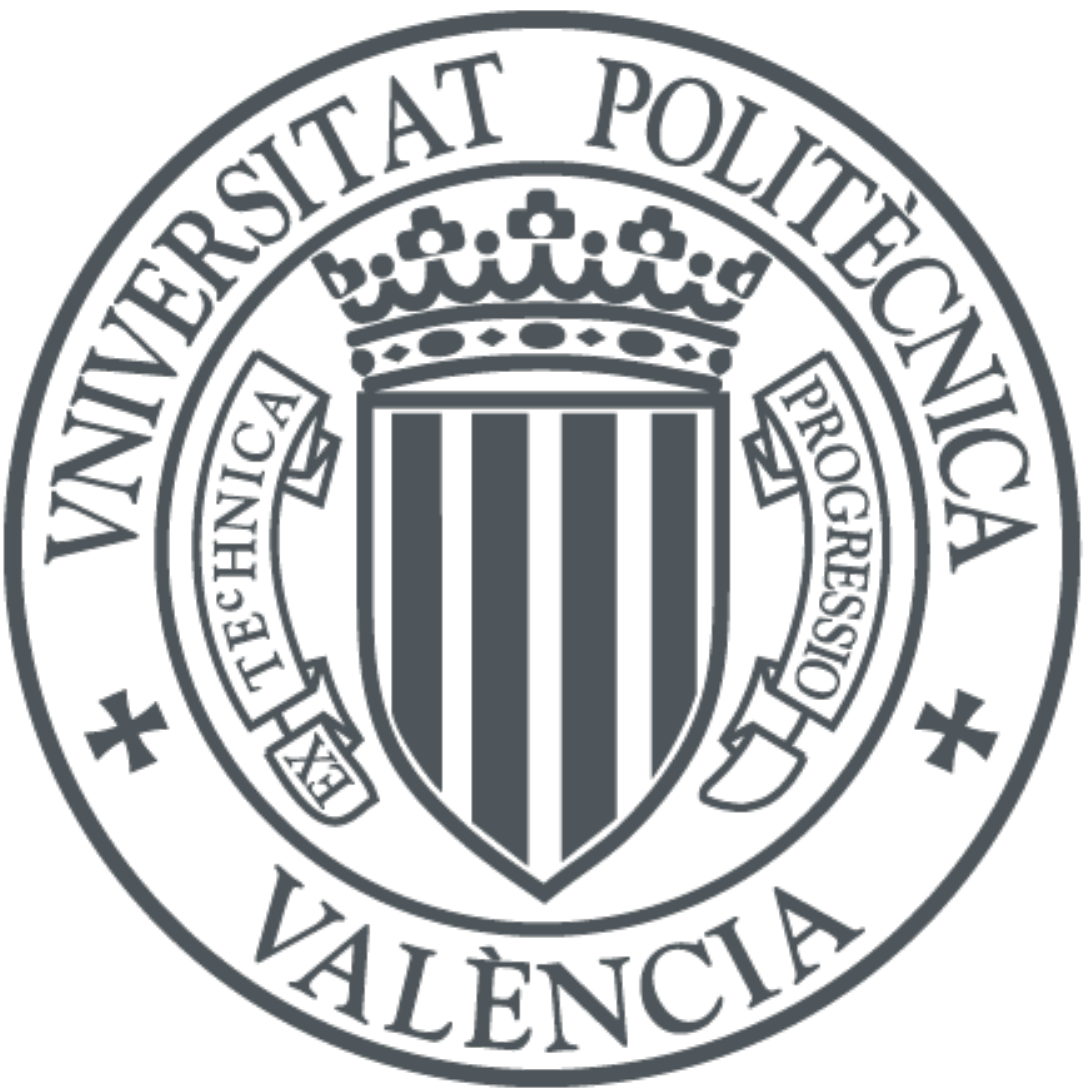

The final publication is available at

http://dx.doi.org/10.1002/cem.2585

Copyright Wiley

Additional Information 


\title{
Prostate functional Magnetic Resonance Image Analysis using Multivariate Curve Resolution methods
}

José Manuel Prats-Montalbán ${ }^{\mathrm{a}}$, Roberto Sanz-Requena ${ }^{\mathrm{b}}$, Luis Martí-Bonmatín ${ }^{\mathrm{b}, \mathrm{c}}$, Alberto Ferrer $^{\mathrm{a}}$

a) Multivariate Statistical Engineering Group, Applied Statistics Department, Universitat Politècnica de València, Cno. De Vera s/n, Edificio 7A, 46022, Valencia, Spain

b) Radiology Department, Hospital Quirón Valencia, Avda. Blasco Ibáñez 14, 46010, Valencia, Spain

c) Medical Imaging Area, Hospital Universitari i Politècnic La Fe, Bulevar Sur s/n, 46026, Valencia, Spain

\begin{abstract}
This paper discusses the potential of Multivariate Curve Resolution (MCR) models to extract physiological dynamics behaviors from Dynamic Contrast Enhanced Magnetic Resonance (DCE-MR) Imaging prostate perfusion studies for cancer diagnosis. A relationship with biomarkers ("hidden" parameters for assessing the possible existence of a tumor) from pharmacokinetic models is also studied.
\end{abstract}


Keywords: Multivariate image analysis, MCR, SIMPLISMA, EFA, DCE-MR, pharmacokinetics, perfusion, prostate, tumor, biomarker.

\section{INTRODUCTION}

Angiogenesis and neovascularization are biological processes associated to tissues with increases oxygen and nutrients demands. These processes seldom occur in healthy subjects but they are strongly present in pathological conditions such as tumors. The increase in blood perfusion due to the formation of new and tortuous vessels can be studied with dynamic contrast-enhanced magnetic resonance (DCE-MR) imaging [1]. In these studies, an exogenous contrast media is administered intravenously and diffuses from the capillary network into the extravascular extracellular space, establishing a dynamic relationship between image signal intensity changes and the amount of contrast media that passes and diffuses into a certain tissue. The capability to analyze tumor angiogenesis in a quantitative and reproducible way from DCE-MR images has important applications to depict and gradate tumors, and also to evaluate the therapeutic response early after treatment onset [2-3].

To obtain quantitative measurements it is necessary to characterize the intensity vs. time curves associated to each pixel of the image. Out of the different approaches proposed to achieve this evaluation, mathematical pharmacokinetic models have become the most popular in recent years due to their ability to provide physiologically meaningful parameters [4]. 
One of the critical steps in pharmacokinetic modeling is the selection of an appropriate arterial input function (AIF) obtained from the tumor-feeding artery [5-8]. This function is a time-varying curve with a specific dynamics pattern, comprising a baseline, an abrupt positive peak and a fast decay or washout (Figure 1a). The determination of this curve is often based on the visual inspection of a volume of images and the manual selection of the region of interest (ROI) including the artery. This approach introduces a user-dependent bias into the analysis, penalizing the reproducibility of the results.

One characteristic of these models is the lack of a priori knowledge about the tissue vascular environment, which leads to a series of assumptions that condition the use of different pharmacokinetic modeling approaches [9]. Accordingly, and depending on the tissue dynamics patterns (Figure 1), the pharmacokinetic model may provide biased measurements which may not properly reflect the true physiology of the tissue [10]. Therefore, having a priori knowledge about the tissue dynamics may help to interpret the information provided by the pharmacokinetic parameters.

\section{[INSERT FIG. 1 ABOUT HERE]}

This variability affecting the calculations and interpretation of the results provided by these parametric models has limited their applicability and widespread use in clinical practice, as it is difficult to obtain a validation and standardization of the parameters that ensure reproducibility among different studies.

One possible way to look for these dynamic behaviors is by applying multivariate statistical projection models to the DCE-MR imaging data. In image analysis, this application is known as Multivariate Image Analysis (MIA) [11-12], usually based on Principal Component Analysis (PCA) [13]. Its application to oncology [14] allows 
extracting the sources of variation from a relevant number of time-sequenced images from different individuals, providing new statistical models that help explaining the perfusion differences between healthy and cancer affected tissues. MIA has also been applied with segmentation and classification purposes by Gurjal et al. [15], as well as for segregating between healthy and diseased livers [16]; showing the potentials of the technique.

By using MIA, the multivariate model might even better describe the dynamics than the mathematical imposed model, better pointing out to the pixels of interest for improving the model. This would may be at least partially due to the fact that "the wealth of physiological and pharmacokinetic assumptions likely oversimplifies an exceptionally complex system [14]", varying from patient to patient. Thus, this procedure comes out as a proper way to deal with this natural variation and seek for the best candidate pixels for the estimation of the AIF, thus reducing the uncertainty in the estimation of the pharmacokinetic parameters [17-18].

The drawback of the application of PCA in DCE-MR image analysis is that the estimated dynamics patterns are orthogonal by design. Since real dynamics do not need to be orthogonal this is an important limitation of this approach. There exist other chemometric models, such as the Multivariate Curve Resolution (MCR) models [19-20] which do not impose these restrictions. Applications of MCR models on biomedical images can be found in [21], where the score images obtained from these models as well as from PCA scores from near-IR FT-Raman images are used for segmentation purposes on rabbit kidney calculi.

The aim of this paper is to evaluate the applicability of MCR to DCE-MR imaging of prostate and to compare the extracted dynamic components with the quantitative parameters (i.e. biomarkers) estimated from the generalized pharmacokinetic model. 
In Section 2, the materials and methods used are presented. Section 3 shows the extracted dynamics for one case and compares the results from the MCR-based proposed methodology to those obtained from the pharmacokinetic models. Finally, conclusions are outlined in Section 4.

\section{MATERIALS AND METHODS}

\subsection{Image acquisition}

Ten patients with surgery proven prostate carcinoma were studied with a specific high temporal resolution DCE-MR sequence (3 Tesla magnet, 3D spoiled gradient echo sequence, repetition time $3 \mathrm{~ms}$, echo time $1.7 \mathrm{~ms}$, flip angle $40^{\circ}, 3$ seconds per image, total acquisition time 5'30', 47 non-equally spaced temporal samples, image size 192x192, voxel size $1 \times 1 \times 4 \mathrm{~mm}, 12$ slices covering the whole prostate, contrast agent Gd-DOTA $0.2 \mathrm{ml} / \mathrm{kg}$ injected at $4 \mathrm{ml} / \mathrm{s}$ with $40 \mathrm{ml}$ of saline flush at the same rate). The output of this sequence was a series of 564 images (12 slices x 47 time steps) showing the pelvis area perfusion dynamics after the contrast media administration. All images were anonymized and transferred to a dedicated workstation for post-processing.

\subsection{Pharmacokinetic model}


Pharmacokinetic modeling of DCE-MR images is based on the application of a mathematical analysis to tissue enhancement curves, often on a pixel-by-pixel basis. Here, each individual pixel enhancement curve is fit to the following equation:

$$
C(x, y, t)=C_{A I F}(t) \otimes R(t)
$$

where $C(x, y, t)$ is the tissue enhancement curve at pixel with coordinates $(x, y), C_{A I F}(t)$ is the enhancement curve of the arterial input function (the artery that supplies most blood to the tissue of interest), $R(t)$ is the tissue response function and $\otimes$ is the convolution operator.

It is usually assumed that:

$$
R(t)=K^{\text {trans }} e^{-k_{e p} t}
$$

where $\mathrm{k}_{\mathrm{ep}}$ is the washout constant, and $\mathrm{K}^{\text {trans }}$ is the transfer constant.

The generalized kinetic model can be expressed as [4]:

$$
C(x, y, t)=K^{\text {trans }} \int_{0}^{t} C_{A I F}(\tau) e^{-k_{e p} \tau} d \tau
$$

The model needs as input the reference arterial input function $\left(\mathrm{C}_{\mathrm{AIF}}\right)$ and the tissue enhancement curves $C(x, y, t)$. These curves are usually extracted by manually placing ROIs in the artery (i.e. one of the iliac arteries for the prostate) and the tissue of interest (i.e. the prostate in this study). Then the pharmacokinetic parameters $\mathrm{k}_{\mathrm{ep}}$ and $\mathrm{K}^{\text {trans }}$ can be obtained by curve fitting algorithms for each pixel in the image area under study. This model was applied to the time-sequenced images obtained for each of the 10 patients analyzed.

\subsection{Multivariate Curve Resolution models}


For each slice of the studied tissue, DCE-MR images can be interpreted as a 3D data volume formed by a $2 \mathrm{D}$ image obtained at each time point $t$. This $2 \mathrm{D}$ matrix is built by the $n_{1} \times n_{2}$ pixels that form a gray level image for each channel in the time dimension. For simplicity, in this study only one central slice including the prostate is analyzed for each patient.

Once the 3D $\underline{\mathbf{X}}\left(n_{1} \times n_{2} \times T\right)$ image is defined, the following step is to take each of the $T$ channels (i.e. time points) and unfold the $n_{1} \times n_{2}$ pixels into a column, thus forming a 2D $\mathbf{X}\left(\left(n_{1} \times n_{2}\right) \times T\right)$ matrix that can be analyzed by MCR.

In this paper, a Multivariate Curve Resolution-Alternating Least Squares (MCR-ALS) [19-20, 22-23] model has been used because of its ability to provide physically more interpretable results by imposing some a priori knowledge on the model and the lack of orthogonality restriction on the internal relationships between variables (extracted dynamics). The idea behind MCR, traditionally applied in analytical chemistry, can be easily expanded to the concept of dynamic perfusion phenomena, stating that the pixel enhancement curve $\mathrm{C}(x, y, t)$ is a linear combination of the different "pure" dynamic behaviors existing in it. The lack of orthogonality restriction in the pure dynamic behaviors improves the model in terms of physiological interpretability.

MCR-ALS is an iterative method that performs a bilinear decomposition of matrix $\mathbf{X}$ by means of an alternating least squares optimization:

$\mathbf{X}=\mathbf{C S}^{\mathrm{T}}+\mathbf{E}$

In this particular application, $\mathbf{S}^{\mathrm{T}}$ is a matrix containing in its rows each one of the dynamic behaviors modeled, $\mathbf{C}$ gathers the relative importance of each modeled dynamic behavior for each pixel of the image, and $\mathbf{E}$ is a residual matrix. 
MCR relies in the correct determination of the number of "real" dynamic behaviors in a data set (the image in this case). When some a priori information is available, it can be used as an initial estimation. In this case, when a tumor is evolving in the prostate, three different dynamic behaviors can be expected:

1. Type A: drastic enhancement and fast washout (AIF) (blood flowing within the arteries), corresponding to the dynamics pattern in the artery (Fig. 1a).

2. Type NT: low progressive enhancement without washout, corresponding to a non-tumoral tissue (Fig. 1b).

3. Type VT: delayed drastic enhancement and widened washout in comparison to the perfusion in the arteries, corresponding to a highly vascular tissue, such as a tumor (Fig. 1c),

This a priori knowledge can be checked by using some tool able to show up the relevant sources of information in the time-sequenced images. One possible way to do this is by applying PCA on the data set, and taking a look at the number of latent variables (principal components, PC's) with the highest variance. Once the number of likely dynamic behaviors present in the image is determined, the purest dynamics in the raw data can be sought using e.g. SIMPLISMA [24, 25] or more recent approaches proposed by Windig [26], ROI's of loadings extracted from PCA (not the best option in this case, because not all the purest dynamic behaviors could be a priori known to be located at some pixels, nor negative dynamics behaviors could happen in reality); genetic algorithms [27-29]; or OPA [30]. In this work, since we have used the software available at the Multivariate Curve Resolution Homepage [31], the SIMPLISMA based algorithm implemented in Pure function has been applied. 
The previous steps only help on the initial estimation of the dynamic behaviors. However, these dynamic behaviors do not need to be present in all pixels. In order to find out the likely number of dynamic behaviors present in a pixel, the Fixed Size Image Window-Evolving Factor Analysis (FSIW-EFA) [32-34] was applied. FSIWEFA performs local PCA models in the whole image by moving small windows around each individual pixel area. This way, the number of relevant PC's (significant singular values) in these small windows advice for the number of constituents (dynamic behaviors) present in that local window. In this work, since it is expected to find three different dynamic behaviors at most at each pixel location, a $2 \times 2$ pixel window was used. One could argue about using a larger window size, because maybe more than four events could be happening in it. However, enlarging the window size necessarily means to lower the spatial resolution of the method, hence loosing precision about the location of the events (dynamic behaviors, then affected tissue), which is critical in these studies. From the number of significant singular values in each pixel area, local rank maps [34] are derived, which inform about the number of different constituents that form each pixel, acting as a constraint map in the MCR model. Depending on the threshold used to separate significant from non-significant singular values (i.e. determining the number of dynamic behaviors at each pixel location), local rank maps may vary. When trying to look for the real dynamics in the image, a good option is to look for pixels with a robust rank estimation. In these cases, partial local rank maps can be used. These maps are obtained after setting a threshold band for significance of eigenvalues. This way, only pixels having a constant rank value between the lowest and the highest limit of the threshold band (i.e. with a more robust rank estimation) appear in this map; leaving the rest unconstrained. Nevertheless, for identification purposes, the local rank information should be combined with reference spectral (dynamic) information, which can be 
approximately obtained by pure variable selection methods, as in this work, or by a previous MCR-ALS analysis with only non-negativity constraints. For further reading, a completely devoted paper to this issue is [35]. Once the number of constituents per pixel has been assessed, it can be imposed as a constraint on the MCR model. Also, two additional constraints were imposed:

1. Non-negativity on the pixel intensity values, because the intensity in a pixel has to be non-negative.

2. Non-negativity on the dynamics profiles.

Regarding the use of additional constraints, as in this case that related to the form of the AIF and its parameterization, since different types of equations might be used [36, 37], we decided not to impose any a priori form that might bias the results, and leave the model free to fit the best AIF in each case. The MCR model built this way provides the matrices $\mathbf{S}^{\mathbf{T}}$ and $\mathbf{C}$ previously commented, gathering the dynamic behaviors found, as well as the corresponding relative importance of each dynamic behavior at each pixel location, respectively. By refolding the $\mathbf{C}$ matrix into the original $n_{1} \times n_{2}$ spatial dimension, new images (known as distribution maps) are obtained, which permit to locate those pixels more related to each of the corresponding dynamic behaviors provided by the model.

The whole sequential procedure is summarized in the following steps:

1. Determination of the approximate number of dynamic behaviors by PCA, according to the number of relevant singular values. If a priori knowledge is available, it should be used.

2. Determination of the pixels with purest dynamics behaviors.

3. Determination of the number of dynamics behaviors present at each pixel location by FSIW-EFA. 
4. Application of the MCR-ALS with the additional constraints of non-negativity both on the pixel intensities and the dynamic behaviors.

Applying these first four steps on the whole pelvic area is necessary in order to obtain an accurate AIF.

5. In order to improve the precision in the prostate area, local MCR models were built for each of the ten prostates analyzed. The same ROIs defined at the pharmacokinetic modeling step were used here for segmentation purposes. The dynamic behaviors obtained in the previous MCR model, with the exception of the AIF (which is present in the arteries, but not in the prostate) can be implemented as initial guess. The same constraints should be imposed.

All calculations for pharmacokinetic modeling and MCR were done using Matlab (The Mathworks Inc., Natick, MA, USA). Multivariate Statistical analyses were performed using SIMCA (MKS Umetrics AB, Umea, Sweden). MCR-ALS algorithms were downloaded from the Multivariate Curve Resolution Homepage [31]

\section{RESULTS}

In order to illustrate the application of the MCR-based methodology on the DCE-MR images one case is discussed in detail. Afterwards, the dynamics obtained from the MCR method on the ten cases studied are compared to the corresponding biomarkers $\mathrm{K}^{\text {trans }}$ and $\mathrm{k}_{\mathrm{ep}}$ estimated from the pharmacokinetic models.

\subsection{MCR analysis}


First step in any MCR analysis on DCE-MR images deals with assessing for the number of significant dynamic behaviors in the image. Figure 2 presents the variances of the first 10 PC's from the fitted PCA model. It can be seen that from PC 5 in advance, all the PC's have similar amount of explained variance, so four significant behaviors can be assumed to be present in the image.

\section{[INSERT FIG. 2 ABOUT HERE]}

Afterwards, the four purest dynamic behaviors to be introduced in the model are found. Figure 3 shows the results corresponding to the case under study for the four dynamic behaviors indicated by PCA.

[INSERT FIG. 3 ABOUT HERE]

The next step consists on applying FSIW-EFA. The results provided about the presence of a different number of dynamic behaviors at each pixel location are presented in Figure 4, related to the abdominal slice containing the prostate analyzed. As can be observed, there are some internal parts of the abdominal slice that have zero value, which actually means that the model is free for providing the number of dynamics behaviors at those pixel locations. In fact, the external parts surrounding the abdomen, which are just background, were extracted from the model for computational and noise removal purposes.

[INSERT FIG. 4 ABOUT HERE] 
Using this partial local rank map as one constraint, as well as the non-negativity on the pixels' intensities and on the dynamic behaviors in the MCR model, matrices $\mathbf{C}$ and $\mathbf{S}$, gathering the relative importance of each dynamic behavior at each pixel location and the dynamic behaviors, respectively, are provided. Figure 5 shows the obtained dynamic behaviors.

\section{[INSERT FIG. 5 ABOUT HERE]}

In order to visualize where these dynamic behaviors are more predominant, it is necessary to refold each of the columns of the $\mathbf{C}$ matrix into their original spatial dimension, $n_{1} \times n_{2}$, obtaining the dynamic behaviors distribution maps (Figure 6). By inspecting both figures, we can appreciate that VT behavior (Figs. 5 and 6, bottom left) seems to reproduce the perfusion in the arteries (type A) (Figs. 5 and 6, top left), but delayed around 2 time steps, and with a bit more of amplitude. This behavior is happening in some internal area of the prostate, and it is an indicator of a highly vascularized region. In addition, Fig. 6, top left, provides the pixels that show the arterial perfusion process sought, shown in Fig. 5 top left, corresponding to the iliac arteries. Furthermore, there is a progressive enhancement in the rest of the prostate, which is in accordance to the healthy-like contrast uptake process (Figs. 5 and 6, top right). Finally, there is an additional non-physiological contrast media arrival (type CMA) effect (Figs. 5 and 6, bottom right) of no physiological interest, but also captured by the model. This non-desired effect is probably inherent to MR studies, which use an exogenous contrast media. The arrival of the contrast media in the tissue of interest changes its magnetic properties and the signal intensity, so that the images are slightly affected too, though the MR equipment usually auto-scales them to minimize the effects. Also, this fourth dynamic is predominant at certain low vascular peripheral 
regions (see Fig. 6 bottom right), which correspond to some specific elements of the MR receiving coil.

\section{[INSERT FIG. 6 ABOUT HERE]}

Up to this step, PCA was also tested in the 10 cases under study (results not shown). It was also able to extract out the AIF function, but not the segregation between the behaviors in the prostate, nor the existence of the type CMA behavior. Furthermore, the fact of imposing orthogonality often forced parts of the AIF to disaggregate on more than one PC.

\subsection{Local MCR prostate modeling}

The dynamic behaviors previously detected may still be estimated more precisely when focusing into the prostate area. In order to deal with this issue, MCR local models for the prostate area were fitted. This approach dismissed the AIF pure arterial behavior, since it is not present in the prostate region of interest. Figures 7a) shows the final optimized dynamic behaviors and distribution maps for the prostate area MCR local model. Furthermore, MCR bands [38] were also applied in order to check for possible ambiguities (Fig 7b), and study the feasibility of the final dynamic behaviors obtained. In all 10 cases, the bands were very close to the proposed solution. Only in one case the CMA dynamic behavior presented one bound different and separated from the proposed one. Nevertheless, the CMA proposed dynamic behavior and its upper bound were validated by the doctors as the realistic ones, as they were similar to the rest of cases. 


\section{[INSERT FIG. 8 ABOUT HERE]}

As can be seen from comparison with Fig. 5 (bottom left), the highly vascular tissue dynamic behavior (blue in Fig. 7a), bottom-left in Fig. 5) has changed significantly, although in a way that keeps the same delay (the peak is also located at the 15th time step) and larger amplitude. The healthy-like enhancing tissue (red in Fig 7a), top-right in Fig. 5) and the contrast media arrival effect (green in Fig 7a), bottom right in Fig. 5) remain essentially the same. From Fig. 8, it can be observed how, even still present, the non-physiological contrast media arrival effect has almost disappeared, and only the healthy-like tissue keeps similar intensity values to the highly vascular tissue dynamic.

\subsection{Dynamics from MCR versus pharmacokinetic biomarkers}

The MCR analysis was performed for all the 10 cases under study, obtaining the relative importance of each of the three dynamic behaviors found for the MCR local models, once the AIF was extracted from the global models. In addition, the pharmacokinetic model (Eq. 3, Section 2.2) was applied to each of the 10 patients analyzed, obtaining the estimated biomarkers $\mathrm{K}^{\text {trans }}$ and $\mathrm{k}_{\mathrm{ep}}$ for each one of the pixels of the patient prostate images under study.

Pharmacokinetic modeling is a highly time consuming task requiring expert knowledge. On the other hand, MCR analysis is rather fast and easy to apply. Therefore, it is worth to study the relationship between the dynamic behaviors (type VT, NT and CMA) obtained by MCR-ALS and the estimated pharmacokinetic biomarkers ( $\mathrm{K}^{\text {trans }}$ and $\left.\mathrm{k}_{\mathrm{ep}}\right)$. Two PLS-1 models, one with $\mathrm{K}^{\text {trans }}$ and the other with $\mathrm{k}_{\mathrm{ep}}$ as $\mathbf{y}$ response variable, were 
fitted. The $\mathbf{X}$ matrix was built with the prostate area pixels from the ten cases analyzed; stacking each patient one below the other (i.e. pixels from patient 1, then pixels from patient 2, etc.). For each pixel, distribution maps values for types VT, NT and CMA dynamics were registered as $\mathbf{X}$ variables. Moreover, in order to estimate not only overall but also patient specific relationships, cross-products effects (i.e. interaction terms) between dynamic behaviors and cases (i.e. patients) were modeled. In both PLS-1 models interactions between types NT and CMA with the cases did not provide statistically significant coefficients in general ( $p$-values $>0.05$ ), so they are not included in the final PLS models, for simplicity considerations.

Figure 9 shows the coefficients plot with $95 \%$ jackknife confidence intervals from the $\mathrm{K}^{\text {trans }}$ PLS-1 model built, (goodness of prediction $\mathrm{Q}^{2}=0.83$ ). The first three coefficients (VT, NT and CMA) show the overall linear relationship (average for all the patients studied) of the three dynamic behaviors with $\mathrm{K}^{\text {trans }}$. The following ten coefficients (Case (1), Case (10)...Case (9)) model $\mathrm{K}^{\text {trans }}$ average differences between the ten patients studied. The last ten coefficients (VT*Case (1), VT*Case (10)...VT*Case (9)) estimate the slope difference of the relationship for each particular patient with respect to the overall linear relationship modeled by VT coefficient.

\section{[INSERT FIG. 9 ABOUT HERE]}

From Figure 9, there is a statistically significant high overall positive linear relationship of type VT dynamics with $\mathrm{K}^{\text {trans }}$, which is not statistically related to type NT in general, and has a small negative relationship with type CMA dynamics. There are also statistically significant patient specific effects not only in terms of average $\mathrm{K}^{\text {trans }}$ values ( 
see case(\#) coefficients) but also on different slopes in the relationship of $\mathrm{K}^{\text {trans }}$ with type VT dynamics for each patient (see VT*case(\#) coefficients). Given the fact that the interaction coefficients are smaller than the overall linear effect (VT coefficient), the

relationship between $\mathrm{K}^{\text {trans }}$ with type VT dynamics is positive for all the patients, although with a different value. This means that, although with a different intensity depending on each patient, the highly vascular tissue dynamic behavior, characteristic of some tumors, is positive related with the transfer constant, $\mathrm{K}^{\text {trans }}$, biomarker. Figure 10 shows the predicted $v s$ observed values for $\mathrm{K}^{\text {trans }}$ for the 10 cases analyzed.

\section{[INSERT FIG. 10 ABOUT HERE]}

Regarding washout constant, $\mathrm{k}_{\mathrm{ep}}$, biomarker, similar results have been obtained for the PLS-1 model built, with a goodness of prediction $\mathrm{Q}^{2}=0.75$ (see Figures 11 and 12). From Fig. 11, which presents the coefficients plot with $95 \%$ confidence intervals, again it is possible to see the main statistically significant overall positive relationship of type VT, with a small overall negative effect of type NT is this case, as well as for type CMA. Also again, depending on the case studied, different average values and positive slopes of $\mathrm{k}_{\mathrm{ep}} v s$ type VT are expected.

[INSERT FIG. 11 ABOUT HERE]

[INSERT FIG. 12 ABOUT HERE] 
These results reflect the heterogeneous dynamic behaviors associated to tumors, where several enhancement patterns may coexist and interact at each pixel and with particularities for each patient.

\section{CONCLUSIONS}

In this work, MCR was used instead of PCA in MIA to extract physiological dynamic behaviors in the prostate from DCE-MR images, as real dynamics do not need to be orthogonal and probably they are not. Also, MCR allows introducing constraints about non-negativity, which is not possible when using PCA, because of the orthogonality restrictions; as well as about the number of dynamic behaviors (components) present in a pixel.

The obtained results provide optimized dynamics related to different enhancement patterns, such as the AIF (type A dynamics), healthy-like tissues (type NT dynamics) and highly vascularized tissues (type VT dynamics). Apart from the expected dynamics, a fourth dynamic behavior was obtained corresponding to the contrast media arrival (type CMA dynamics). Further studies using different MR acquisition sequences and equipment are necessary to assess the influence of this effect. In fact, the MCR workflow presented in this study could be used to subtract the influence of nonphysiological dynamic effects, should they be biasing the calculation of the pharmacokinetic parameters. 
The successful extraction of the AIF can be potentially used to set an automatic procedure that locates the nearest AIF to the tissue of interest, as is the prostate in this case. Therefore, the bias associated to the manual selection of the AIF (needed in pharmacokinetic modeling) would be eliminated and a more reproducible pharmacokinetic analysis would be achieved [5,17].

Furthermore, by using final local (prostate) MCR models, in all cases it has been possible to segregate the highly vascular (VT) and normal-enhancing (NT) dynamics in the prostate, establishing their relationship with both $\mathrm{K}^{\text {trans }}$ and $\mathrm{k}_{\mathrm{ep}}$ constants through PLS models, which indicate a clear positive linear relationship with type VT and a dependence on the patient.

This relationship has a potential interest to disaggregate pixel-by-pixel the transfer constant $\mathrm{K}^{\text {trans }}$ into perfusion (highly-vascular dynamics) and permeability (normal enhancement pattern) components [10], which is a current issue in pharmacokinetic modeling of DCE-MRI studies, especially in the analysis of tumors where areas with very different vascular behaviors coexist.

Therefore, these PLS models can finally be used on a patient basis as a fast method to characterize the highly vascular and normal dynamics contributions to the pharmacokinetic model biomarkers. Further studies using these dynamic behaviors are required to assess for the precision and benefits of the proposed methodology.

\section{ACKNOWLEDGEMENTS}


This research work was partially supported by the Spanish Ministry of Economy and

Competitiveness under the project DPI2011-28112-C04-02.

\section{REFERENCES}

1. Collins DJ, Padhani AR. Dynamic magnetic resonance imaging of tumor perfusion. IEEE Eng Med Biol Mag 2004; 23, 65-83.

2. Jackson ASN, Reinsberg SA, Sohaib SA, et al. Dynamic contrast-enhanced MRI for prostate cancer localization. Br J Radiol 2009; 82,148-156.

3. Leach MO, Brindle KM, Evelhoch JL, et al. The assessment of antiangiogenic and antivascular therapies in early-stage clinical trials using magnetic resonance imaging: issues and recommendations. Br J Cancer 2005; 92,1599-1610.

4. Tofts PS, Brix G, Buckley DL, et al. Estimating kinetic parameters from dynamic contrast-enhanced T1-weighted MRI of a diffusable tracer: standardized quantities and symbols. J Magn Reson Imaging 1999; 10, 223-232.

5. Port RE, Knopp MV, Brix G. Dynamic contrast-enhanced MRI using Gd-DTPA: interindividual variability of the arterial input function and consequences for the assessment of kinetics in tumors. Magn Reson Med 2001; 45, 1030-1038.

6. McGrath DM, Bradley DP, Tessier JL, et al. Comparison of model-based arterial input functions for dynamic contrast-enhanced MRI in tumor bearing rats. Magn Reson Med 2009; 61, 1173-1184.

7. Yang C, Karczmar GS, Medved M, et al. Reproducibility assessment of a multiple reference tissue method for quantitative dynamic contrast-enhanced MRI analysis. Magn Reson Med 2009; 61, 851-859. 
8. Meng R, Chang SD, Jones EC, et al. Comparison between population average and experimentally measured arterial input function in predicting biopsy results in prostate cancer. Acad Radiol 2010; 17, 520-525.

9. Sourbron SP, Buckley DL. Tracer kinetic modelling in MRI: estimating perfusion and capillary permeability. Pys Med Biol 2012; 57, 1-33.

10. Lüdemann L, Prochnow D, Rohlfing T, et al. Simultaneous quantification of perfusion and permeability in the prostate using dynamic contrast-enhanced magnetic resonance imaging with an inversion-prepared dual-constrast sequence. Ann Biomed Eng 2009; 37, 749-762.

11. Geladi P, and Grahn H. Multivariate Image Analysis. Wiley. Chichester, England, 1996.

12. Prats-Montalbán JM, Ferrer A, and de Juan A Multivariate Image Analysis: a review with applications. Chemom. Intell. Lab. Syst. 2011; 107, 1-23.

13. Jackson J E. A User's guide to Principal Components. Ed. Wiley, New York, 1991.

14. Bruwer MJ, MacGregor JF, Noseworthy D. Dinamic contrast-enhanced MRI diagnosis in oncology via principal component analysis. J. Chemometrics 2008; 22, 708-716.

15. Gujral P, Amrhein M, Bonvin D, Vallée JP, Montet X, Michoux N. Classification of magnetic resonance images from rabbit renal perfusion. Chemom. Intell. Lab. Syst. 2009, 98, 173-181.

16. Fortuna J, Elzibak AH, Fan Z, MacGregor JF, Noseworthy MD. Liver functional magnetic resonance imaging analysis using a latent variable approach. J. of Chemometrics 2012, 26, 170-179.

17. Buckley DL. Uncertainty in the analysis of tracer kinetics using dynamic contrastenhanced T1-weighted MRI. Magn Reson Med 2002; 47, 601-606. 
18. Henderson E, Sykes J, Drost D, Weinmann H-J, Rutt BK, Lee T-Y. Simultaneous MRI measurement of blood flow, blood volume, and capillary permeability in mammary tumours using two different contrast agents. J Magn Reson Imaging 2000; 12, 991-1003.

19. Tauler R, Smilde AK, Kowalski BR. Selectivity, local rank, three- way data analysis and ambiguity in multivariate curve resolution. J. Chemometrics. 1995; 9, 31-58.

20. Tauler R. Multivariate curve resolution applied to second order data. Chemom. Intell. Lab. Syst 1995; 30, 133-146.

21. Piqueras S, Duponchel L, Tauler R, de Juan A. Resolution and segmentation of hyperspectral biomedical images by Multivariate Curve Resolution-Alternating Least Squares. Anal. Chim. Acta 2011; 705, 182-192

22. de Juan A, Tauler R. Chemometrics applied to unravel multicomponent processes and mixtures: Revisiting latest trends in multivariate resolution. Anal. Chim. Acta $2003 ; \mathbf{5 0 0}, 195-210$.

23. Jaumot J, Gargallo R, de Juan A, Tauler R. A graphical user-friendly interface for MCR-ALS: a new tool for multivariate curve resolution in MATLAB Chemom. Intell. Lab. Syst. 2005; 76, 101-110.

24. Windig W, Guilment J. Interactive self-modeling mixture analysis. Anal. Chem. $1991 ; 63,1425-1432$.

25. Gallagher NB, Shaver JM, Martin EB, Morris J, Wise BM, Windig W. Curve resolution for multivariate images with applications to TOF-SIMS and Raman. Chemom. Intell. Lab. Syst. 2004; 73, 105-117. 
26. Windig W. Two-way data analysis, detection of Purest Variables. In Comprehensive Chemometrics (Brown, Tauler and Walczak eds.), Vol. 2, Elsevier, 2009, 275-307.

27. Leardi R (ed.), Nature-inspired Methods in Chemometrics: Genetic Algorithms and Artificial Neural Networks, in Data Handling in Science and Technology Vol.23, Amsterdam, Elsevier, 2003.

28. Chtioui Y, Bertrand D, Barba D. Feature selection by a genetic algorithm. Application to seed discrimination by artificial vision. J. Sci. Food Agric. 1998; 76, 77-86.

29. Wang M, Zhou XB, King EW, Wong STC. BMC Bioinform. 2007; 8, 32.

30. Cuesta Sánchez F, Toft J, van den Bogaert B, Massart DL. Orthogonal Projection Approach applied to Peak Purity Assessment. Anal. Chem. 1996; 68, 79-85

31. Multivariate Curve Resolution Homepage, http://www.mcrals.info/.

32. de Juan A, Maeder M, Hancewicz T, Tauler R. Local rank analysis for exploratory spectroscopic image analysis. Fixed size image window-evolving factor analysis. Chemom. Intell. Lab. Syst. 2005; 77 (1-2), 64-74.

33. Ked HR, Massart DL. Peak purity control in liquid chromatography with photodiode-array detection by a fixed size moving window evolving factor analysis Anal. Chim. Acta 1991; 246, 379-390.

34. de Juan A, Maeder M, Hancewicz T, Duponchel L, Tauler R, Chemometric tools for image analysis, Infrared and Raman Spectroscopic Imaging, Wiley-VCH Verlag GmbH \& Co. KGaA, 2009, 65-109.

35. de Juan A, Maeder M, Hancewicz T, Tauler R, Use of local rank-based spatial information for resolution of spectroscopic images. J. Chemometrics 2008; 22, 291298. 
36. Orton MR, d'Arcy JA, Walker-Samuel S, Hawkes DJ, Atkinson D, Collins DJ, Leach MO. Computationally efficient vascular input function models for quantitative kinetic modelling using DCE-MRI. Physics in Medicine and Biology 2008; 53, 1225-1239

37. Parker GJM, Roberts C; Macdonald A, Buonaccorsi GA, Cheung S, Buckley DL, Jackson A, Watson Y, Davies K, Jayson GC. Experimentally-Derived Functional Form for a Population-Averaged High-Temporal-Resolution Arterial Input Function for Dynamic Contrast-Enhanced MRI. Magn Reson Med 2006; 56, 993-1000

38. Jaumot J, Tauler R. MCR-BANDS: A user friendly MATLAB program for the evaluation of rotation ambiguities in Multivariate Curve Resolution. Chemom. Intell. Lab. Syst. 2010; 103, 96-107. 